\title{
Two glyoxylate reductase isoforms are functionally redundant but required under high photorespiration conditions in rice
}

\author{
Zhisheng Zhang ${ }^{1,2}$, Xiu Liang ${ }^{1,2}$, Lei Lu ${ }^{1,2}$, Zheng Xu ${ }^{1,2}$, Jiayu Huang ${ }^{1,2}, H_{a n} \mathrm{He}^{3}$ and Xinxiang Peng ${ }^{1,2^{*}}$
}

\begin{abstract}
Background: The glyoxylate reductase (GR) multigene family has been described in various plant species, their isoforms show different biochemical features in plants. However, few studies have addressed the biological roles of GR isozymes, especially for rice.

Results: Here, we report a detailed analysis of the enzymatic properties and physiological roles of OsGR1 and OsGR2 in rice. The results showed that both enzymes prefer NADPH to NADH as cofactor, and the NADPHdependent glyoxylate reducing activity represents the major GR activity in various tissues and at different growth stages; and OsGR1 proteins were more abundant than OsGR2, which is also a major contributor to total GR activities. By generating and characterizing various OsGR-genetically modified rice lines, including overexpression, single and double-knockout lines, we found that no phenotypic differences occur among the various transgenic lines under normal growth conditions, while a dwarfish growth phenotype was noticed under photorespirationpromoted conditions.
\end{abstract}

Conclusion: Our results suggest that OsGR1 and OsGR2, with distinct enzymatic characteristics, function redundantly in detoxifying glyoxylate in rice plants under normal growth conditions, whereas both are simultaneously required under high photorespiration conditions.

Keywords: Glyoxylate reductase, Glyoxylate, Photorespiration, Oxalate, Rice

\section{Background}

Plant glyoxylate reductase (GR; EC 1.1.1.26/79) is a key enzyme involved in aldehydes metabolism, which catalyzes the reduction of glyoxylate and succinic semialdehyde (SSA) to glycolate and $\gamma$-hydroxybutyrate using $\mathrm{NAD}(\mathrm{P}) \mathrm{H}$ as a cofactor $[1,2]$. GR activity was first detected in crude extracts of spinach leaves. Subsequently, GR was successfully purified from tobacco and spinach

\footnotetext{
* Correspondence: xpeng@scau.edu.cn

${ }^{1}$ State Key Laboratory for Conservation and Utilization of Subtropical Agro-bioresources, College of Life Sciences, South China Agricultural University, Guangzhou, China

${ }^{2}$ Guangdong Laboratory for Lingnan Modern Agricultural Science and Technology, South China Agricultural University, Guangzhou, China Full list of author information is available at the end of the article
}

leaves; both of which prefer NADPH to NADH as cofactor [3-5]. Later on, two GR isozymes were molecularly identified in Arabidopsis thaliana, further analysis confirmed that AtGR1 (At3g25530) is localized to the cytosol while AtGR2 (At1g17650) is localized in plastids and mitochondria [6]. Recently, with NADPH as cofactor, the catalytic characteristics of GR isozymes from apple, rice and A.thaliana were analyzed, showing that glyoxylate $\left(K_{m}=19.1 \sim 53.2 \mu \mathrm{M}\right)$ is preferred over SSA $\left(K_{m}=\right.$ $870 \sim 8960 \mu \mathrm{M})$ as substrate for all GR isoforms [6, 7].

Previous evidences found that expression of GR genes was upregulated under abiotic stresses (e.g. salinity, drought and submergence), accompanied by the accumulation of $\gamma$-hydroxybutyrate, suggesting that GR is involved in the detoxification of SSA in response to abiotic 
stresses [8,9]. Zarei et al. [7] showed that in the presence of exogenous glyoxylate, AtGR-RNAi lines are more sensitive to chilling stress than wild type (WT), and characterization of $A t G R$-overexpression lines further support that glyoxylate reduction catalyzed by AtGRs is closely related to stress resistance of A. thaliana. It is noteworthy that a major source for glyoxylate production is photorespiratory metabolism in plants, where glycolate is oxidized to glyoxylate by glycolate oxidase (GLO) [10-12]. Generally, photorespiratory glyoxylate is quickly detoxified by serine:glyoxylate aminotransferase and glutamate:glyoxylate aminotransferase in peroxisomes, but it has been considered that the glyoxylate leaking from peroxisomes under photorespiration-promoting conditions needs to be scavenged by GRs $[10,11,13]$. However, no molecular genetic evidences have been presented so far to prove this opinion. In addition, glyoxylate is an efficient precursor for oxalate biosynthesis in plants [14], and various functions have been reported for oxalate, including heavy metal detoxification, ion balance, pathogen defense and tissue support $[15,16]$. It has been noticed that oxalate regulation does not necessarily depends on photorespiratory rates $[14,17]$, which may be due to some other anaplerotic pathways that have participated in glyoxylate regulation $[7,14,18,19]$. Hence, oxalate generation from glyoxylate is also likely to be regulated in a complex manner, and it has not yet been reported whether GR mediates the regulation of glyoxylate-dependent oxalate accumulation.

To date, GR genes were identified in various plant species [20], but only the GR isoforms from A.thaliana were molecularly and biochemically characterized $[6,20]$. The function and mechanism of GRs are still largely unknown, and particularly few studies addressed in vivo roles of GRs in crop plants. By performing BLAST with the sequences of AtGR1 and AtGR2, two GR homologs in the rice genome were identified (i.e. OsGR1-Os02g0562700 and OsGR2-Os01g0574600). Here, to understand the catalytic properties and physiological roles related to glyoxylate metabolism, OsGR1 and OsGR2 were biochemically and genetically investigated. The results showed that the two OsGR isozymes, localized in the cytosol or chloroplasts, had distinct biochemical and enzymatical properties. Further, we generated various genetically modified rice lines for both $O s G R$ genes. There were no phenotypic differences noticed for single or double OsGR mutants under normal natural conditions and even no differences were observed for either single mutant under high photorespiration conditions, whereas a stunted growth was noticed only for the OsGR1/OsGR2 double mutants under high photorespiration conditions. Taken together, our results demonstrate that the two OsGR isoforms, with distinct enzymatic characteristics, are functionally redundant but both are simultaneouly required under high photorespiration conditions in rice.

\section{Results}

Expression patterns and subcellular localization of OsGR1 and OsGR2

Up to now, AtGR1 and AtGR2 are most extensively studied among all of the GRs from different plants $[6,8,9]$. The rice genome contains two GR homologs: OsGR1 located on chromosome 2, and OsGR2 seated on chromosome 1. The similarity between OsGR1, OsGR2, AtGR1 and $A t G R 2$ is appreciably high (Table 1, Additional file 1). Both OsGR1 and OsGR2 may use $\mathrm{NAD}(\mathrm{P}) \mathrm{H}$ as cofactor, so we detected the total $\mathrm{NAD}(\mathrm{P}) \mathrm{H}$-dependent GR activities in rice leaves separately. The NADPH-dependent activity was much higher than the NADH-dependent activity, both of which showed a fluctuation throughout the day (Fig. 1a). Besides, GR activities displayed variation during different growth stages, being highest at the vigorous vegetative stage (Fig. 1b). Transcript abundances of the two $O s G R$ genes were determined by real-time quantitative PCR (qRT-PCR). As shown in Fig. 1c, OsGR1 displayed much higher transcriptional levels than OsGR2 in all organs tested. Moreover, OsGR1 was abundant in leaves and glume, and moderately expressed in other organs, whereas OsGR2 was expressed primarily in leaf sheaths, glume and leaves. Proteomic data suggested that the protein abundance ratio of OsGR1 to OsGR2 ranged from 1.2 to 1.4 in leaves (Table 2). The results indicate that both OsGR1 and OsGR2 are widely distributed in photoautotrophic tissues, and their NADPH-dependent activities contribute mostly to total GR activities in rice.

Subcellular localization of OsGR1 and OsGR2 was first predicted by the WoLF PSORT and Plant-mPLoc [21, 22], turning out that OsGR1 could be a cytoplasmic protein and OsGR2 likely located in the chloroplast. To experimentally prove the prediction, OsGR1 and OsGR2 fused with green fluorescence protein (GFP) were transiently expressed in rice protoplasts. As shown in Fig. 1d, OsGR1-GFP was observed to localize in the cytosol, while OsGR2-GFP in the chloroplast. Generally, chloroplast proteins are synthesized in cytosol, with an N-terminal chloroplast transit peptide (CTP) linked as a precursor form; the precursor form is guided into chloroplast by the CTP, which is then proteolytically removed after import

Table 1 Similarities of OsGR and AtGR isoforms at the level of protein and nucleotide

\begin{tabular}{lllll}
\hline $\begin{array}{l}\text { Protein } \\
\text { mRNA }\end{array}$ & OsGR1 & OsGR2 & AtGR1 & AtGR2 \\
\hline OsGR1 & & & & \\
OsGR2 & $61.7 \%$ & $58.1 \%$ & $80.7 \%$ & $58.2 \%$ \\
AtGR1 & $58.7 \%$ & $49.3 \%$ & & \\
AtGR2 & $58.4 \%$ & $56.5 \%$ & $45.6 \%$ & $58.2 \%$ \\
\hline
\end{tabular}

Lipman-Pearson method was used for protein sequence alignment, WilburLipman method was used for mRNA sequence alignment 


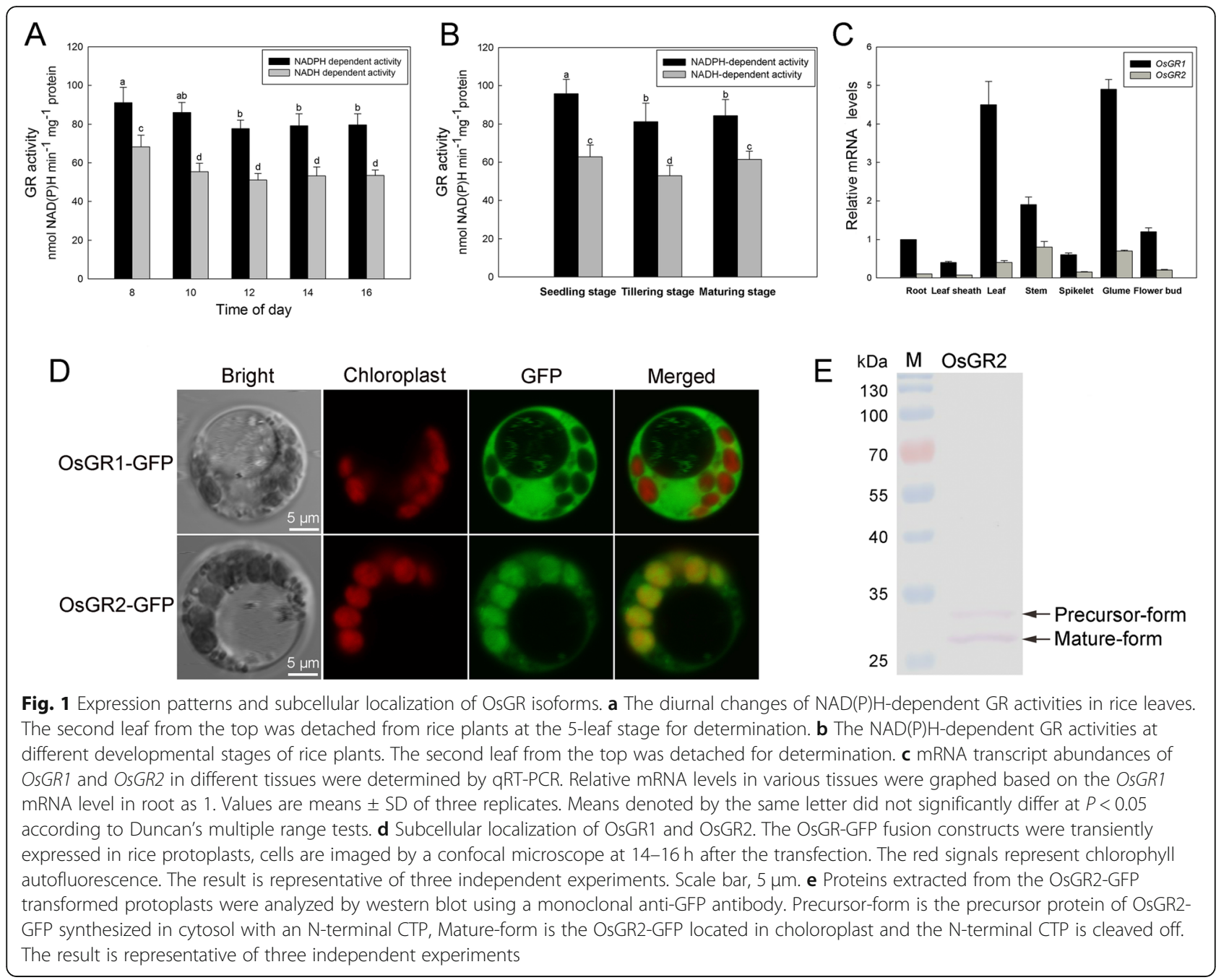

into the chloroplast [23, 24]. Proteins extracted from the rice protoplasts transiently expressing the OsGR2-GFP were used for western blot assay with anti-GFP antibody. The results showed that a large number of OsGR2-GFP proteins were detected as mature form without CTP (Fig. 1e), further proving that OsGR2 was localized in the chloroplast.

\section{Enzymatic characterization of OsGR1 and OsGR2}

There are few studies about enzymatic properties of the OsGR isozymes. A preliminary research has been recently made on NADPH kinetics of OsGR1 and OsGR2 [7]. To further explore functions of OsGR1 and OsGR2, we comparatively analyzed glyoxylate-dependent enzymatic differences of the two OsGR isoforms. A $6 \times$ His-tag was fused to a full-length OsGR1 and a truncated OsGR2 sequence (without N-terminal CTP), then these OsGR sequences were cloned into pColdIV vector and expressed in E.coli. The OsGR1 and OsGR2 proteins were subsequently purified from the E.coli crude extract by His-tag affinity chromatography. SDS-PAGE analysis showed that molecular weights of OsGR1 and OsGR2 subunits were approximately $30 \mathrm{kDa}$ and $37 \mathrm{kDa}$, respectively (Fig. 2a); similar optimum $\mathrm{pH}$ in the range of 7.0-7.5 was observed for OsGR1 and OsGR2 (Fig. 2b). The kinetic analyses were performed at physiological temperature $\left(30^{\circ} \mathrm{C}\right)$ and $\mathrm{pH}$ 7.4. As shown in Table 3,

Table 2 Protein relative abundances of OsGR1 and OsGR2 in WT detected by proteomic analysis

\begin{tabular}{llllll}
\hline Proteins & Acesssion & Coverage [\%] & $\begin{array}{l}\text { Unique } \\
\text { Peptides }\end{array}$ & $\begin{array}{l}\text { Abundances (Normalized) } \\
\text { Sample 1 }\end{array}$ & $\begin{array}{l}\text { Abundances (Normalizd) } \\
\text { Sample 2 }\end{array}$ \\
\hline OSGR1 & Q84VC8 & 57 & 13 & 668.1 & 648.5 \\
OSGR2 & Q656T5 & 46 & 9 & 561.3 & 475
\end{tabular}

Total proteins from three independent WT plants (Sample 1, 2 and 3) were used for proteomic analysis 
OsGR1 and OsGR2 exhibited a much higher affinity for NADPH than for NADH. The glyoxylate-linked $K_{m \text { (NADPH) }}$ values of OsGR1 and OsGR2 were 17.6 and $53 \mu \mathrm{M}$, while the $K_{m(\mathrm{NADH})}$ values were 420.2 and $403.9 \mu \mathrm{M}$, respectively. The $\mathrm{NAD}(\mathrm{P}) \mathrm{H}$-dependent kinetics with glyoxylate for the two OsGRs were also determined. The NADPH-linked $K_{m \text { (glyoxylate) }}$ values for OsGR1 and OsGR2 were 30.4 and $72.1 \mu \mathrm{M}$, and the NADH-linked $K_{m \text { (glyoxylate) }}$ values were 267.7 and $144.6 \mu \mathrm{M}$ (Table 3), respectively. Besides, the $V_{\max }$ of OsGR1 with NAD(P) $\mathrm{H}$ were higher than that of OsGR2. It is noteworthy that glyoxylate is an efficient precursor for oxalate biosynthesis in plants, and rice leaves contain high levels of oxalate ranging from 25 to $120 \mathrm{mM}$ [14, 17, 25]. Therefore, we tested if oxalate had a feedback effect on the glyoxylate reduction catalyzed by OsGRs. As shown in Table 4, when using NADPH as cofactor, the $K_{i}$ value of oxalate for OsGR1 was $21.2 \mathrm{mM}$ and that for OsGR2 was $290.8 \mathrm{mM}$. When using NADH as cofactor, the $K_{i}$ values of oxalate were much lower, $3.6 \mathrm{mM}$ for OsGR1, and $8.2 \mathrm{mM}$ for OsGR2. The above results indicated that OsGR1 and OsGR2 have high affinity for glyoxylate, and both prefer $\mathrm{NADPH}$ to NADH as cofactor, with the NADH-dependent activity much more sensitive to oxalate inhibition than the NADPH-dependent activity.

\section{Characterization of OsGR-genetically modified plants}

Thus far, few data could be found on the biological roles of OsGR isoforms, particularly for rice. In this study, the open reading frames of OsGR1 and OsGR2 were used to construct the OsGR-overexpression transgenic lines, the knockout lines were generated by CRISPR-Cas9 system, and the T2 heterozygous lines were used for the analysis here. As expected, in leaves of OsGR-overexpression
(OX-OsGR) lines each $O s G R$ gene was up-regulated at mRNA levels (Additional file 2), and correspondingly, the GR activities were elevated by $5-8$-folds in OXOsGR1 lines and 1-3-folds in OX-OsGR2 lines (Fig. 3a). When OsGR1 and OsGR2 were knocked out using pYLCRISPR/Cas9 system (Additional files 2 and 3), GR activities were decreased by $35-45 \%$ and $20-25 \%$ in leaves of OsGR1 and OsGR2 knockout mutants, respectively, indicating that OsGR1 is the major contributor to total GR activities (Fig. 3a). In the OsGR1/OsGR2 double mutant, the GR activities dropped by $60-75 \%$ (Fig. 3a). The residual activities might come from some other enzymes that were reported to reduce glyoxylate as a side reaction, such as hydroxypyruvate reductases (HPR) $[5,26]$. Subsequently, glyoxylate levels in these genetically modified rice lines were analysed. Under normal growth conditions, the glyoxylate content of WT was about $6.5 \mu \mathrm{g}$ $\mathrm{g}^{-1} \mathrm{FW}$, and increased by $0.4-0.8 \mu \mathrm{g} \mathrm{g}^{-1} \mathrm{FW}$ in OsGR1 and OsGR2 knockout mutants, and $1.2-1.5{\mu \mathrm{gg}^{-1}} \mathrm{FW}$ in OsGR1/OsGR2 double mutant, while no obvious decreases in glyoxylate contents were observed in OXOsGR lines (Fig. 3b). Moreover, all plants accumulated much more glyoxylate under high photorespiration conditions, with WT being increased to approximately $18.0 \mathrm{\mu g} \mathrm{g}^{-1} \mathrm{FW}$, and OsGR1/OsGR2 double mutant up to $28.0 \mu \mathrm{g} \mathrm{g}^{-1} \mathrm{FW}$ (Fig. 3b). In addition, oxalate levels of these rice lines under normal growth conditions were measured. The oxalate contents increased ranging from 20 to $25 \%$ in the OsGR1 and OsGR2 single knockout mutants, and from 30 to $40 \%$ in the OsGR1/OsGR2 double mutants (Fig. 3c). No changes were observed in both OX-OsGR1 and OX-OsGR2 lines (Fig. 3c).

Under normal conditions, these OsGR-genetically modified plants showed no significant phenotypic
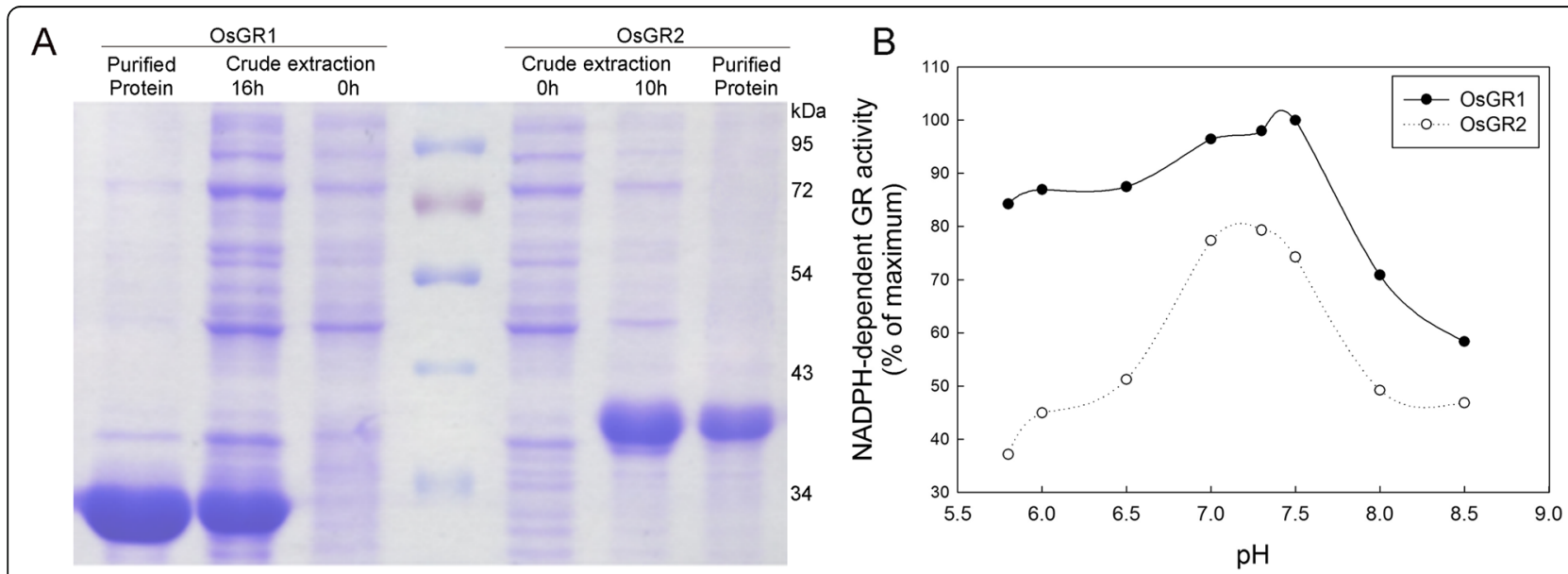

Fig. 2 Expression of OsGR isoforms in E.coli. a OsGR1 and OsGR2 were expressed in E.coli cells and then purified using immobilized metal affinity chromatography, their purity and subunit molecular weights were determined by SDS-PAGE. The results are representative of three independent experiments. $\mathbf{b}$ Effect of $\mathrm{pH}$ on the NADPH-dependent activities of OsGR1 and OsGR2. The activities were detected at a physiological temperature $\left(30^{\circ} \mathrm{C}\right)$, and activities were expressed as a percentage of the maximum value, each point represents the mean of three determinations 
Table $3 K_{m}$ and $V_{\max }$ of OsGR1 and OsGR2 with various substrates

\begin{tabular}{|c|c|c|c|c|}
\hline Enzyme & Substrate at fixed concentration & Substrate at varying concentration & $K_{m}(\mu \mathrm{M})$ & $V_{\max }\left(\mu \mathrm{mol}\right.$ glyoxylate $\mathrm{min}^{-1} \mathrm{mg}^{-1}$ protein $)$ \\
\hline \multirow[t]{4}{*}{ OsGR1 } & Glyoxylate & $\mathrm{NADPH}$ & $17.6 \pm 3.1$ & $74.7 \pm 5.9$ \\
\hline & $\mathrm{NADPH}$ & Glyoxylate & $30.4 \pm 3.5$ & \\
\hline & Glyoxylate & $\mathrm{NADH}$ & $420.2 \pm 50.5$ & $59.9 \pm 8.1$ \\
\hline & $\mathrm{NADH}$ & Glyoxylate & $267.7 \pm 22.7$ & \\
\hline \multirow[t]{4}{*}{ OsGR2 } & Glyoxylate & $\mathrm{NADPH}$ & $53.0 \pm 4.6$ & $61.9 \pm 3.2$ \\
\hline & $\mathrm{NADPH}$ & Glyoxylate & $72.1 \pm 5.3$ & \\
\hline & Glyoxylate & $\mathrm{NADH}$ & $403.9 \pm 66.5$ & $22.4 \pm 3.5$ \\
\hline & $\mathrm{NADH}$ & Glyoxylate & $144.6 \pm 19.3$ & \\
\hline
\end{tabular}

The OsGR1 and OsGR2 expressed in E.coli were purified for analyses, and $K_{m}$ values with different substrate pairs were compared. Values are means \pm SD ( $n=3$ )

differences as compared with WT (Fig. 4a). However, when the photorespiration was promoted by low $\mathrm{CO}_{2}$ concentration and continuous light [27, 28], the OsGR1/ OsGR2 double mutants showed stunted growth (Fig. 4b, Additional file 4). The OX-OsGR1 and OX-OsGR2 lines displayed no apparent phenotypic differences under both normal and photorespiration-promoted conditions (data not shown). These results stronly support that OsGR1 and OsGR2 are involved in glyoxylate detoxification under certian stressful conditions which stimulate photorespiration, although both may function redundantly at most of the time.

\section{Discussion}

Biological roles of different gene members in multigene family could be diverse in general [29-33]. The GR multigene family exists in various plant species, such as Arabidopsis, Sinorhizobium meliloti, apple and rice [2, 7], and their GR isoforms display different expression patterns, biochemical features and functions. It has been reported that GR might be involved in the detoxification of glyoxylate and succinic semialdehyde in Arabidopsis and tobacco under certain stressfull conditions $[1,6]$. Zarei et al. [7] further demonstrated that the NADPH/ $\mathrm{NADH}$ ratio might regulate the expression of GR in Arabidopsis and mediate their roles in the aldehydes detoxification. However, by reviewing the literature, little detailed information is available about the physiological roles of GR in rice.

Here, we first show that the NADPH-dependent glyoxylate reducing activity is the major GR activity in different tissues and at different growth stages of rice (Fig. 1a,

Table $4 K_{i}$ of OsGR1 and OsGR2 with oxalate

\begin{tabular}{lll}
\hline Enzyme & $\begin{array}{l}K_{\text {i(oxalate) }} \\
(\mathrm{mM})\end{array}$ & \\
\cline { 2 - 3 } & $\begin{array}{l}\text { Glyoxylate as substrate } \\
\text { NADPH as cofactor }\end{array}$ & $\begin{array}{l}\text { Glyoxylate as substrate } \\
\text { NADH as cofactor }\end{array}$ \\
\hline OsGR1 & $21.2 \pm 4.3$ & $3.6 \pm 0.8$ \\
OsGR2 & $290.8 \pm 59.7$ & $8.2 \pm 1.1$ \\
\hline
\end{tabular}

Values are means $\pm \mathrm{SD}(n=3)$ b), similar to the GR from spinach and tobacco [4, 34]. Further, analyses at transcription levels and protein abundance suggest that OsGR1 is the major GR isoform in rice (Fig. 1c, Table 2). Subcellular localization of GR remains controversial, and recent research demonstrated that the Arabidopsis AtGR1 was localized in cytoplasm, while AtGR2 was localized in plastids and mitochondria $[7,20]$. In the present study, confocal microscopy observation localized OsGR1 in the cytoplasm, while OsGR2 in the chloroplast (Fig. 1d), and western blot assay further proved that OsGR2 is a chloroplastic protein (Fig. 1e).

Determing enzymatic properties is a conventional way to indirectly reveal biological functions of enzymes [29, 35, 36]. GR proteins were first purified from tobacco and spinach leaves and then biochemically and enzymatically characterized, showing that the glyoxylate-linked $K_{m(\mathrm{NADPH})}$ values of GR from tobacco and spinach are 0.32 and $0.13 \mathrm{mM}$, respectively [4]. GR isoforms from Arabidopsis, apple and rice showed catalytic activities not only to reduce glyoxylate but also to reduce succinic semialdehyde [7]. In this study, glyoxylate-dependent enzymatic properties of OsGR1 and OsGR2 were comparatively studied. OsGR1 and OsGR2 showed the highest activities in the physiological $\mathrm{pH}$ range (7.0-7.5) (Fig. 2b), and both prefer NADPH to NADH as cofactor (Table 3), consistent with GR isozymes from other species $[2,4,6]$. As shown in Table 3, the NADPH-linked $K_{m \text { (glyoxylate) }}$ of OsGR1 was $30.2 \mu \mathrm{M}$, significantly lower than that of OsGR2 $(72.1 \mu \mathrm{M})$, indicating that OsGR1 has a higher affinity for glyoxylate than OsGR2. Nevertheless, the physiological concentrations of glyoxylate in plants range from 100 to $300 \mu \mathrm{M}[17,18,37]$, which are higher than the NADPH-linked $K_{m \text { (glyoxylate) }}$ values of OsGR1 and OsGR2. Therefore, both OsGR1 and OsGR2 may efficiently catalyze the reduction of glyoxylate at physiological concentrations in rice. In addition, rice is an oxalate-accumulating plant, oxalate contents in rice range from 20 to $100 \mathrm{mM}[14,25]$. Based on the $K_{i \text { (oxalate) }}$ of OsGR1 and OsGR2 (Table 4), under physiological conditions, the endogenous oxalate might be able to partially suppress the NADPH-dependent glyoxylate reducing activity of OsGR1, but not OsGR2, as a result, it is likely that in vivo OsGR1 and OsGR2 may work in a similar 

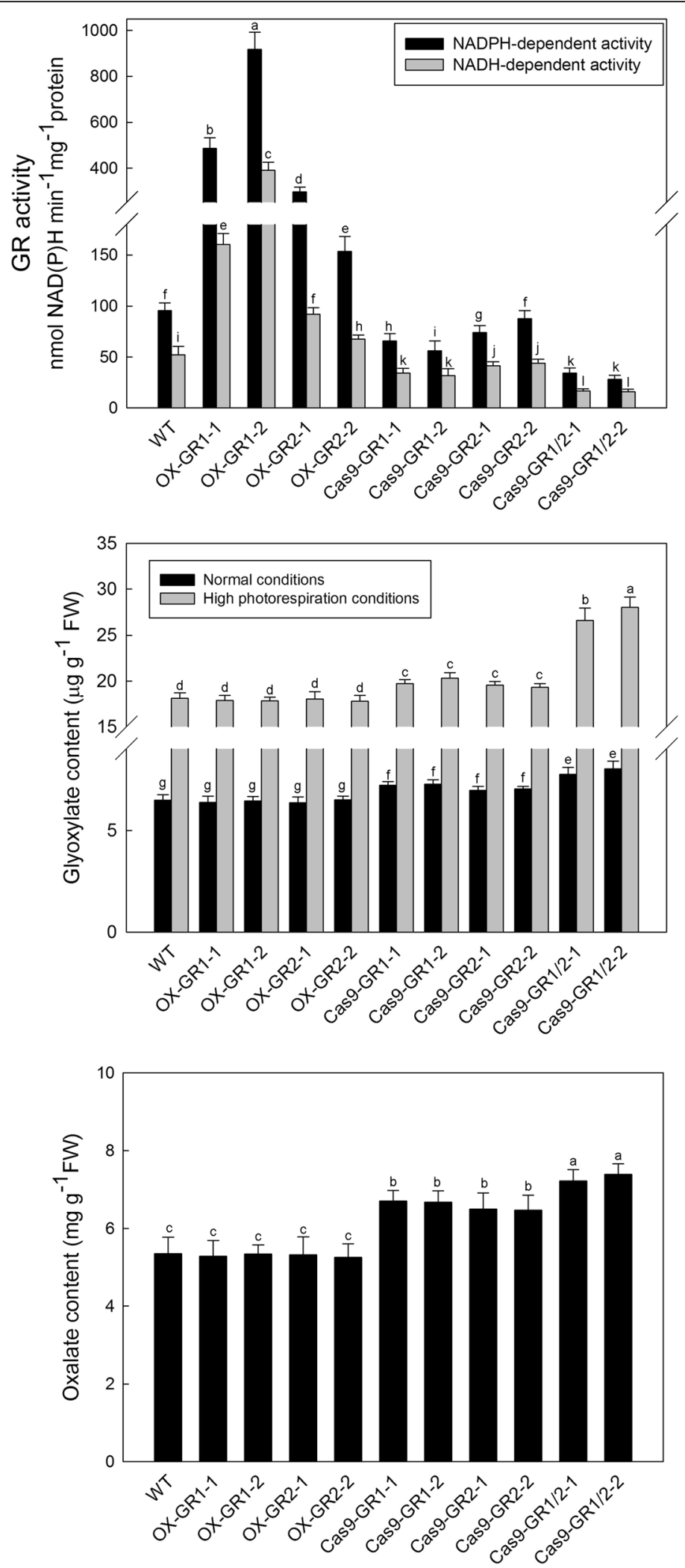

Fig. 3 (See legend on next page.) 
(See figure on previous page.)

Fig. 3 Catalytic characteristics of OsGR isoforms in vivo. a NAD(P)H-dependent glyoxylate reducing activities in different OsGR-genetically modified plants. $\mathbf{b}$ The accumulation of glyoxylate in different OsGR-genetically modified plants under normal growth conditions and high photorespiration conditions. c The accumulation of oxalate in different OsGR-genetically modified plants under normal growth conditions. OXGR1 and OX-GR2 represent the OsGR1 and OsGR2 overexpression plants; Cas9-GR1 and Cas9-GR2 represent the OsGR1 and OsGR2 single knockout mutants; Cas9-GR1/2 represent the OsGR1 and OsGR2 double knockout mutants. Values are means \pm SD of three replicates. Means denoted by the same letter did not significantly differ at $P<0.05$ according to Duncan's multiple range tests

efficient manner to reduce glyoxylate. However, the NADHdependent glyoxylate reducing activity of OsGR1 and OsGR2 could be inhibited by the physiological concentrations of oxalate, such that the NADH-dependent activities of GR may have not actually functioned in the glyoxylate detoxification in rice plants.

Photorespiratory glyoxylate is produced in peroxisomes and usually accumulated under high photorespiration or other stressful conditions, which is highly toxic to plant cells if not metabolized or removed timely [38, 39]. Moreover, glyoxylate is a small organic acid, which can diffuse from peroxisomes to other cellular compartments [11, 19, 40-44]. Previous studies assumed that photorespiratory glyoxylate escaping from peroxisomes could be scavenged by GRs, but direct molecular genetic evidences are still scarce $[10,11,13]$. In order to further verify if OsGR1 and OsGR2 function in the glyoxylate detoxification in vivo, various $O s G R$-genetically modified rice lines were generated and the glyoxylate contents were detected in those transgenic plants. It was noticed that the WT and OX-OsGR lines had same levels of glyoxylate even under high photorespiration conditions (Fig. 3b), likely because the background GR activities in WT are high enough to remove the photorespiratory glyoxylate leaking from peroxisome, so that further overexpression would be needless. Previous studies have also reported that there are certain anaplerotic reactions that compensate for the loss of glyoxylate in plants $[14,18,19,38]$. Nevertheless, the glyoxylate levels were increased in the $O s G R$ knockout mutants, particularly in the double mutants (Fig. 3b), and this value became much higher under high photorespiration conditions (Fig. 3b). Unexpectedly, however, neither their single nor the double mutants showed phenotypic changes under normal conditions (Fig. 4a, c), negative phenotypes could be noticed for the double mutant only under high photorespiration conditions (Fig. 4b, c and Additional file 4). This implicates that the glyoxylate accumulation due to deficiency of OsGR1 and OsGR2 might have not yet attained the toxic threshold for rice plants under normal conditions, and the two OsGR isoforms are both required for efficiently removing glyoxylate under high photorespiration conditions (e.g. drought, heat, high light). Overall, the datas stronly support that OsGR1 and OsGR2 are indeed involved in glyoxylate detoxification under certian stressfull conditions that stimulate photorespiration, although both may function redundantly sometimes. In addition, oxalate contents of various $G R$ - genetically modified rice lines were also analyzed. As seen from the Fig. 3c, along with the glyoxylate increase of OsGR knockout mutants (Fig. 3b), oxalate was increased by $25-30 \%$ in both the OsGR1 and OsGR2 single mutants, and by $30-40 \%$ in the OsGR1/OsGR2 double mutants. These results further confirmed our previous conclusion that glyoxylate is an efficient precursor for oxalate biosynthesis, and both OsGR1 and OsGR2 may be involved in the regulation of oxalate metabolism in rice. Molecular mechanisms of these biological processes remain obscure and await further investigation.

\section{Conclusions}

GR activities not only show interdiurnal variations, but also fluctuate during different growth stages. OsGR1 is a cytoplasmic protein, while OsGR2 is localized in the chloroplast. OsGR1 contributes more to total GR activities, and has a higher affinity for glyoxylate than OsGR2, although both can efficiently catalyze the reduction of glyoxylate using NADPH as preferred cofactor in rice plants. Under normal conditions, OsGR1 and OsGR2 are involved in the detoxification of glyoxylate in a nonobligatory manner, likely because the accumulated glyoxylate due to deficiency of OsGR1 and OsGR2 may have not attained the toxic threshold for rice plants under normal conditions. Nevertheless, the two OsGR isoforms are simultaneously required to cope with the over-production of glyoxylate under high photorespiration conditions.

\section{Methods}

\section{Plant materials and growth conditions}

Oryza sativa cv. Zhonghua 11 (japonica cultivar-group) preserved by our laboratory was used for generation of the transgenic lines and the functional analyses $(\mathrm{Xu}$ et al., 2006). Pre-germinated rice seeds were normally grown in KimuraB complete nutrient solution [45] under greenhouse condition ( $14 \mathrm{~h}$ light/10 h dark, average temperature of $30 / 25^{\circ} \mathrm{C}$ (light/dark), relative humidity $60-80 \%$ and average light intensity of $600 \mu \mathrm{mol} \mathrm{m}^{-2}$ $\mathrm{s}^{-1}$ ). In order to promote photorespiration, the rice plants were cultivated in enclosed glass tubes $(21 \mathrm{~cm}$ height, $3.0 \mathrm{~cm}$ diameter) under continuous light, and then the $\mathrm{CO}_{2}$ levels in the tubes decreased rapidly, facilitating the flux of the photorespiration [27, 28]. 


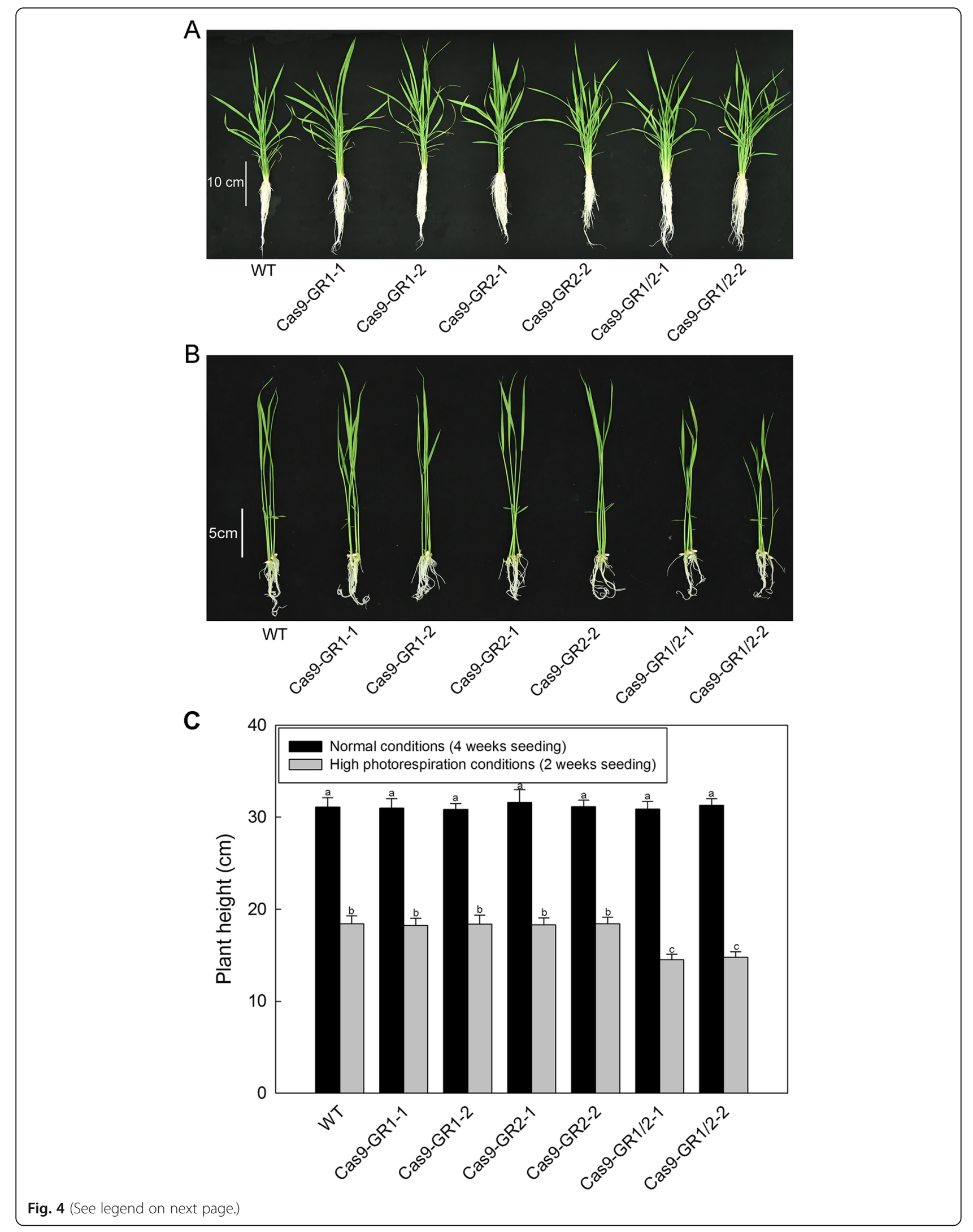


(See figure on previous page.)

Fig. 4 Growth phenotypes of the OsGR-knockout mutants. a Phenotypes of the OsGR-knockout mutants growth under normal condition (4 weeks seedling). Scale bar, $10 \mathrm{~cm}$. b Phenotypes of the OsGR-knockout mutants growth under photorespiration-promoted conditions. $5 \mathrm{Scale}$ bar, $5 \mathrm{~cm}(2$ weeks seedling). c Plant height. Cas9-GR1 and Cas9-GR2 represent the OsGR1 and OsGR2 single knockout mutants; Cas9-GR1/2 represent the OsGR1 and OsGR2 double knockout mutants. Values are means \pm SD of three replicates. Means denoted by the same letter did not significantly differ at $P<0.05$ according to Duncan's multiple range tests

\section{Plasmid construction}

Total RNA was extracted from rice leaves using EZ-10 Total RNA Mini-Preps Kit (Sangon Biotech, China), and the purified RNA was assessed with a NanoPhotometer (IMPLEN, Germany). First-strand cDNA was synthesized using HiScript 1st Strand cDNA Synthesis Kit (Vazyme, China). Primers were designed to include the complete open reading frames of two GR genes. To generate GRoverexpression transgenic lines, each GR sequence was cloned into pYLox.5 vector using ClonExpress Ultra One Step Cloning Kit (Vazyme, China). For the generation of CRISPR-Cas9 knockout lines, specific targeting sequences of OsGR1 and OsGR2 (Additional file 3) were synthesized and cloned into pYLCRISPR/Cas9Pubi vector [46]. To construct the vectors for protein heterologous expression in E.coli, the full-length OsGR1 and the truncated OsGR2 (without N-terminal CTP) sequences were cloned into pColdI vector. The sequence of the first $879 \mathrm{bp}$ of the OsGR1 and the first $153 \mathrm{bp}$ of the OsGR2 were used to generate the OsGR1-GFP-pBI121 and OsGR2-GFPpBI121vectors [33].

\section{RNA isolation and qRT-PCR}

First-strand cDNA was synthesized using HiScript 1st Strand cDNA Synthesis Kit as above (Vazyme, China). The specific primer pairs were designed for the qRTPCR of OsGR1 and OsGR2 (Additional file 5). The qRTPCR reaction mixture consisted of $0.2 \mu \mathrm{M}$ (each) primer, $10 \mu \mathrm{L}$ of $2 \times$ SYBR Green PCR Master Mix (Toyobo, Japan), and $2 \mu \mathrm{L}$ of appropriate diluted cDNA. The analysis was conducted using a DNA Engine Opticon 2 Real-Time PCR Detection system and Opticon Monitor software (Bio-Rad, CA). The data were normalized to the amplification of the OsActin1 gene (Os03g0718100).

\section{Quantitative proteomic analysis}

Protein extracts were obtained according to $\mathrm{Lu}$ et al. (2013) with some modifications [38]. Crude protein extracts were obtained by grinding $1.0 \mathrm{~g}$ rice leaves in liquid nitrogen followed by resuspension in $5 \mathrm{ml}$ ice-cold Tris- $\mathrm{HCl}(100 \mathrm{mM}, \mathrm{pH} 8.5)$ containing $6 \mathrm{M}$ guanidine chloride $(\mathrm{GdnHCl})$ and $1 \%$ protease inhibitor cocktail (Abcam, USA), protein amount was quantified by BCA assay (Thermo Fisher Scientific, USA). Subsequently, $200 \mu \mathrm{g}$ of lysate was added to four volumes of methanol followed by an equal volume of chloroform with mixing, three volumes of $\mathrm{ddH}_{2} \mathrm{O}$ were added to the tube with mixing. The solution was centrifuged at $14,000 \mathrm{~g}$ for 5 min. The upper aqueous layer was discarded, the protein pellet was washed with four volumes of methanol, and the tube was centrifuged again. The supernatant was discarded, and the precipitated protein pellet was air-dried. Afterthat, the chromatography-tandem mass spectrometry (LC-MS/MS) analysis was performed by BGI (BGIShenzhen, China).

\section{Generation of genetically modified rice lines}

The various constructed vectors were transformed into rice callus by Agobacterium-mediated infection (strain EHA105) [47]. The T1 seeds from the positive T0 lines were germinated in complete Kimura B nutrient solution, and then transplanted to soil to grow until the T2 seeds were harvested. Afterthat, The T2 heterozygous plants that originated from two independent lines were used for the determination of GR activity and stress treatments.

\section{Expression of proteins in E. coli and their purification}

The OsGR sequences were cloned into pColdI vector, transformed into E. coli Rosseta (DE3) cells and screened on LB-plates containing ampicillin. The proteins were inductively expressed in $E$. coli as previously described with some modifications [48]. Briefly, the transformed $E$. coli cells were incubated with $1 \mathrm{mM}$ of isopropyl-thio- $\beta$ D-1-thiogalactopyranoside (IPTG) at $16^{\circ} \mathrm{C}$ for $10-16 \mathrm{~h}$ with shaking at $180 \mathrm{rpm}$. After that, the supernatant of the $E$. coli cell lysate was purified according to the manufacturer's protocol of Ni-IDA resin (Bio-Rad, USA). The Ni-IDA resin (Bio-Rad) was packed in a Bio-Scale MT5 column $(10 \times 64 \mathrm{~mm})$ up to $2 \mathrm{~mL}$, and then equilibrated with 3 column volumes (CV) of wash buffer (50 $\mathrm{mM}$ PBS, pH 8.0, $10 \mathrm{mM}$ imidazole, $300 \mathrm{mM} \mathrm{NaCl}$ ). The filtered supernatant was prepared by mixed with an equal volume of binding buffer (100 mM PBS, pH 8.0, $20 \mathrm{mM}$ imidazole, $600 \mathrm{mM} \mathrm{NaCl}$ ), and loaded onto the $\mathrm{Ni}$-IDA resin column. The column was washed with 10 $\mathrm{CV}$ of wash buffer, and the bound proteins were eluted with $5 \mathrm{CV}$ of $50 \mathrm{mM}$ PBS (pH 8.0) containing $150 \mathrm{mM}$ imidazole and $300 \mathrm{mM} \mathrm{NaCl}$. The eluted fractions were desalted by ultrafiltration and checked by $12 \%$ SDSPAGE. All the purified proteins were stored in $50 \mathrm{mM}$ PBS ( $\mathrm{pH} 7.0$ ) containing $10 \%$ glycerol at $-75^{\circ} \mathrm{C}$ for subsequent assay. 


\section{GR activity assay}

The GR catalytic activity was monitored as the oxidation of $\operatorname{NAD}(\mathrm{P}) \mathrm{H}$ at physiological temperature $\left(30^{\circ} \mathrm{C}\right)$ according to Zarei et al. [7], unless specific variations were needed in different kinetic and characterization investigations. A typical reaction mixture contains $50 \mathrm{mM}$ PBS (pH 7.4), $0.2 \mathrm{mM} \mathrm{NADPH} \mathrm{(or} 1.0 \mathrm{mM} \mathrm{NADH)}$ ) and 1 $\mathrm{mM}$ glyoxylate. The effects of varying $\mathrm{pH}$ were assayed with $50 \mathrm{mM}$ PBS from pH 5.8 to 8.5. For kinetic parameter determination, catalyses of substrates were performed over a range of concentration $(0.01-0.1 \mathrm{mM}$ NADPH for determining $K_{m(\mathrm{NADPH})}$; $0.1-1.0 \mathrm{mM}$ NADH for determining $K_{m(\mathrm{NADH})}$; $0.01-0.1 \mathrm{mM}$ glyoxylate for determining $K_{m \text { (glyoxylate) }}$ with NADPH as cofactor; 0.1$0.5 \mathrm{mM}$ glyoxylate for determining $K_{m \text { (glyoxylate) }}$ with $\mathrm{NADH}$ as cofactor), the $K_{m}$ was calculated from doublereciprocal plots according to the method of Lineweaver and Burk. Moreover, slopes of the reciprocal plots were then plotted against the concentration of oxalate (10$300 \mathrm{mM}$ with NADPH as cofactor, $2.0-15.0 \mathrm{mM}$ with $\mathrm{NADH}$ as cofactor) to evaluate $K_{i}$ data [49].

\section{Subcellular localization and western blot analysis}

The rice protoplasts were isolated according to Zhang et al. [50], approximate $10 \mu \mathrm{g}$ of GFP-tagged constructs were transfected into $100 \mu \mathrm{L}$ of protoplasts (about $2.0 \times$ $10^{5}$ cells) by PEG-mediated transfection, and then the protoplasts were incubated in the dark at $25^{\circ} \mathrm{C}$ for 14 $16 \mathrm{~h}[50,51]$. The confocal images were captured by a LSCM 780 system (Zeiss, Germany). Proteins extracted from rice protoplasts expressed OsGR2-GFP were separated on $12 \%$ SDS polyacrylamide gel (SDS-PAGE) and electro-blotted onto a nitrocellulose membrane using wet transfer, and the OsGR2-GFP was detected using a GFP-antibody (Abcam, USA).

\section{Determination of glyoxylate and oxalate}

Glyoxylate and oxalate were determined as reported by $\mathrm{Xu}$ et al. [17]. Aliquots of $0.2-0.5 \mathrm{~g}$ leaves, depending on sample availability, were homogenized in $1-4 \mathrm{~mL}$ of 0.5 $\mathrm{N} \mathrm{HCl}$ and heated at $80^{\circ} \mathrm{C}$ for $10 \mathrm{~min}$. After that, the homogenate was added distilled water up to $5-25 \mathrm{~mL}$, and $2-3 \mathrm{~mL}$ of the solution was centrifuged at 12000 rpm for $10 \mathrm{~min}$ and passed through a filter $(0.45 \mu \mathrm{m})$ before HPLC analysis.

Glyoxylate could react with phenylhydrazine to form phenylhydrazone, and then the derivative was quantified by HPLC. Hypsil C18 column $(5 \mu \mathrm{m}, 4.6 \times 250 \mathrm{~mm})$ equipped Waters 550 (Waters, USA) was used as the static phase, a mobile phase containing $5 \%$ methanol and $95 \%$ phosphate buffer (13 $\mathrm{mM}$ potassium biphosphate; $1 \mathrm{mM}$ potassium phosphate dibasic $\mathrm{pH} 6.0$ ) and detection at $324 \mathrm{~nm}$ were applied in this system. For determination of oxalate, the mobile phase was a solution containing $0.5 \% \mathrm{KH}_{2} \mathrm{PO}_{4}$ and $0.5 \mathrm{mM}$ tetrabutylammonium hydrogen sulphate (TBA) buffered at $\mathrm{pH} 2.0$ with orthophosphoric acid, flow rate was $1 \mathrm{~mL} \mathrm{~min}^{-1}$ and detected at $220 \mathrm{~nm}$.

\section{Supplementary information}

Supplementary information accompanies this paper at https://doi.org/10. 1186/s12870-020-02568-0.

Additional file 1. Multiple sequence alignment (MSA) of OsGR and AtGR isoforms at the level of protein and nucleotide.

Additional file 2. OsGR1 and $O s G R 2$ transcript abundances in the leaves of different OsGR-genetically modified plants were determined by qRT$P C R$. Relative $m R N A$ levels in various rice lines were graphed based on the OsGR1 mRNA level in WT as 1.

Additional file 3. Molecular evalution of the Crispr-Cas9 generated OsGR1 and OsGR2 single and double mutants.

Additional file 4. OsGR-knockout mutants growth under photorespiration-promoted conditions. Cas9-GR1 and Cas9-GR2 represent the OsGR1 and OsGR2 single knockout mutants; Cas9-GR1/2 represent the OsGR1 and OsGR2 double knockout mutants. These results are representative of three independent experiments.

Additional file $\mathbf{5}$. The primers used for real-time quantitative PCR.

\section{Abbreviations}

GR: Glyoxylate reductase; SSA: Succinic semialdehyde; CTP: Chloroplast transit peptides; qRT-PCR: Real-time quantitative PCR; GFP: Green fluorescence protein; TBA: Tetrabutylammonium hydrogen sulphate; IPTG: Isopropyl-thio$\beta$-D-1-thiogalactopyranoside

\section{Acknowledgements}

The authors would like to thank Prof. Yao-Guang Liu (College of Life Sciences, South China Agricultural University) for providing the pYLox.5 and PYLCRISPR/Cas9 vectors.

\section{Authors' contributions}

PXX designed the experiments. ZZS, LX and LL performed most of experiments and analyzed the data. XZ, HJY and $\mathrm{HH}$ assisted in experiments. ZZS and PXX wrote the manuscript. All authors read and approved the final manuscript.

\section{Funding}

This work was supported by the Major Program of Guangdong Basic and Applied Research (2019B030302006), the National Natural Science Foundation of China (31770256), and the Guangdong Provincial Natural Science Foundation of China (2019A1515011438). The funding bodies had no role in the design of this study and collection, analysis, and interpretation of data and in writing the manuscript.

\section{Availability of data and materials}

All data generated or analysed during this study are included in this published article and its supplementary information files.

Ethics approval and consent to participate Not applicable.

\section{Consent for publication}

Not applicable.

\section{Competing interests}

The authors declare that they have no competing interests.

\section{Author details}

${ }^{1}$ State Key Laboratory for Conservation and Utilization of Subtropical Agro-bioresources, College of Life Sciences, South China Agricultural University, Guangzhou, China. ${ }^{2}$ Guangdong Laboratory for Lingnan Modern Agricultural Science and Technology, South China Agricultural University, 
Guangzhou, China. ${ }^{3}$ Institute of Fruit Tree Research, Guangdong Academy of Agricultural Sciences, Guangzhou, China.

\section{Received: 10 March 2020 Accepted: 22 July 2020 Published online: 29 July 2020}

\section{References}

1. Allan WL, Clark SM, Hoover GJ, Shelp BJ. Role of plant glyoxylate reductases during stress: a hypothesis. Biochem J. 2009;423(1):15-22.

2. Kutner J, Shabalin IG, Matelska D, Handing KB, Gasiorowska O, Sroka P, Gorna MW, Ginalski K, Wozniak K, Minor W. Structural, biochemical, and evolutionary characterizations of glyoxylate/hydroxypyruvate reductases show their division into two distinct subfamilies. Biochemistry. 2018;57(6): 963-77.

3. Zelitch I, Ochoa S. Oxidation and reduction of glycolic and glyoxylic acids in plants. I. Glycolic acid oxidase. J Biol Chem. 1953;201(2):707-18.

4. Zelitch I, Gotto AM. Properties of a new glyoxylate reductase from leaves. Biochem J. 1962;84(3):541-6.

5. Zelitch I. The isolation and action of crystalline glyoxylic acid reductase from tobacco leaves. J Biol Chem. 1955:216(2):553-75.

6. Simpson JP, Di Leo R, Dhanoa PK, Allan WL, Makhmoudova A, Clark SM, Hoover GJ, Mullen RT, Shelp BJ. Identification and characterization of a plastid-localized Arabidopsis glyoxylate reductase isoform: comparison with a cytosolic isoform and implications for cellular redox homeostasis and aldehyde detoxification. J Exp Bot. 2008;59(9):2545-54.

7. Zarei A, Brikis CJ, Bajwa VS, Chiu GZ, Simpson JP, DeEll JR, Bozzo GG, Shelp BJ. Plant glyoxylate/succinic semialdehyde reductases: comparative biochemical properties, function during chilling stress, and subcellular localization. Front Plant Sci. 2017:8:1399.

8. Allan WL, Simpson JP, Clark SM, Shelp BJ. $\gamma$-hydroxybutyrate accumulation in Arabidopsis and tobacco plants is a general response to abiotic stress: putative regulation by redox balance and glyoxylate reductase isoforms. J Exp Bot. 2008:59(9):2555-64.

9. Allan WL, Breitkreuz KE, Waller JC, Simpson JP, Hoover GJ, Rochon A, Wolyn DJ, Rentsch D, Snedden WA, Shelp BJ. Detoxification of succinate semialdehyde in Arabidopsis glyoxylate reductase and NAD kinase mutants subjected to submergence stress. Botany. 2012;90(1):51-61.

10. Foyer $\mathrm{CH}$, Bloom AJ, Queval G, Noctor G. Photorespiratory metabolism: genes, mutants, energetics, and redox signaling. Annu Rev Plant Biol. 2009; 60(1):455-84.

11. Dellero $Y$, Jossier M, Schmitz J, Maurino VG, Hodges M. Photorespiratory glycolate-glyoxylate metabolism. J Exp Bot. 2016;67(10):3041-52.

12. Zhang Z, Li X, Cui L, Meng S, Ye N, Peng X. Catalytic and functional aspects of different isozymes of glycolate oxidase in rice. BMC Plant Biol. 2017;17(1): 110-35.

13. Bauwe $H$, Hagemann M, Fernie AR. Photorespiration: players, partners and origin. Trends Plant Sci. 2010;15(6):330-6.

14. Yu L, Jiang J, Zhang C, Jiang L, Ye N, Lu Y, Yang G, Liu E, Peng C, He Z. Glyoxylate rather than ascorbate is an efficient precursor for oxalate biosynthesis in rice. J Exp Bot. 2010;61(6):1625-34.

15. Franceschi VR, Nakata PA. Calcium oxalate in plants: formation and function. Annu Rev Plant Biol. 2005;56(1):41-71.

16. Kim KS, Min J, Dickman MB. Oxalic acid is an elicitor of plant programmed cell death during Sclerotinia sclerotiorum disease development. Mol PlantMicrobe Interact. 2008;21(5):605-12

17. Xu H, Ji X, He Z, Shi W, Zhu G, Niu J, Li B, Peng X. Oxalate accumulation and regulation is independent of glycolate oxidase in rice leaves. J Exp Bot. 2006;9(57):1899-908

18. Xu H, Zhang J, Zeng J, Jiang L, Liu E, Peng C, He Z, Peng X. Inducible antisense suppression of glycolate oxidase reveals its strong regulation over photosynthesis in rice. J Exp Bot. 2009;6(60):1799-809.

19. Hoover GJ, Jørgensen R, Rochon A, Bajwa VS, Merrill AR, Shelp BJ. Identification of catalytically important amino acid residues for enzymatic reduction of glyoxylate in plants. BBA Proteins Proteomics. 2013;1834(12): 2663-71.

20. Ching SL, Gidda SK, Rochon A, van Cauwenberghe OR, Shelp BJ, Mullen RT. Glyoxylate reductase isoform 1 is localized in the cytosol and not peroxisomes in plant cells. J Integr Plant Biol. 2012;54(3):152-68.

21. Horton P, Park KJ, Obayashi T, Fujita N, Harada H, Adams-Collier CJ, Nakai K. WoLF PSORT: protein localization predictor. Nucleic Acids Res. 2007:35. W585-7.
22. Chou KC, Shen HB. Cell-PLoc: a package of web servers for predicting subcellular localization of proteins in various organisms. Nat Protoc. 2008; $3(2): 153-62$

23. Shen B, Zhu C, Yao Z, Cui L, Zhang J, Yang C, He Z, Peng X. An optimized transit peptide for effective targeting of diverse foreign proteins into chloroplasts in rice. Sci Rep. 2017:7:46231.

24. Bruce BD. Chloroplast transit peptides: structure, function and evolution. Trends Cell Biol. 2000;10(10):440-7.

25. Ji $X$, Peng $X$. Oxalate accumulation as regulated by nitrogen forms and its relationship to photosynthesis in rice (Oryza sativa L.). J Integr Plant Biol. 2005;47(7):831-8.

26. Givan CV, Kleczkowski LA. The enzymic reduction of glyoxylate and hydroxypyruvate in leaves of higher plants. Plant Physiol. 1992;100(2):552-6.

27. Waszczak C, Kerchev PI, Mühlenbock P, Hoeberichts FA, Katrien VDK Mhamdi A, Willems P, Denecker J, Kumpf RP, Noctor G, Messens J, Breusegem VF. SHORT-ROOT deficiency alleviates the cell death phenotype of the Arabidopsis catalase2 mutant under photorespiration-promoting conditions. Plant Cell. 2016;28(8):1844-59.

28. Chen Z, Kang X, Nie H, Zheng S, Zhang T, Zhou D, Xing G, Sun S. Introduction of exogenous glycolate catabolic pathway can strongly enhances photosynthesis and biomass yield of cucumber grown in a low$\mathrm{CO}_{2}$ environment. Front Plant Sci. 2019;10:702.

29. Ansell $R$, Granath $K$, Thevelein JM, Adler $L$. The two isoenzymes for yeast $\mathrm{NAD}^{+}$-dependent glycerol 3-phosphate dehydrogenase encoded by GPD1 and GPD2 have distinct roles in osmoadaptation and redox regulation. EMBO J. 1997;16(9):2179-87.

30. Odanaka S, Bennett AB, Kanayama Y. Distinct physiological roles of Fructokinase Isozymes revealed by gene-specific suppression of Frk1 and Frk2 expression in tomato. Plant Physiol. 2002;129(3):1119-26.

31. Park SG, Cha M, Jeong W, Kim I. Distinct physiological functions of Thiol peroxidase Isoenzymes in Saccharomyces cerevisiae. J Biol Chem. 2000; 275(8):5723-32.

32. M'rah S, Ouerghi Z, Berthomieu C, Havaux M, Jungas C, Hajji M, Grignon C, Lachaâl M. Effects of $\mathrm{NaCl}$ on the growth, ion accumulation and photosynthetic parameters of Thellungiella halophila. J Plant Physiol. 2006; 163(10):1022-31.

33. Yamagami T, Tsuchisaka A, Yamada K, Haddon WF, Harden LA, Theologis A. Biochemical diversity among the 1-amino-cyclopropane-1-carboxylate synthase isozymes encoded by the Arabidopsis gene family. J Biol Chem. 2004;278(49):49102-12

34. Tolbert NE, Yamazaki RK, Oeser A. Localization and properties of hydroxypyruvate and glyoxylate reductases in spinach leaf particles. J Biol Chem. 1970;245(19):5129-36.

35. Smith $W L$, Langenbach $R$. Why there are two cyclooxygenase isozymes. J Clin Invest. 2001;107(12):1491-5.

36. Escamilla-Treviño LL, Shen H, Uppalapati SR, Ray T, Tang Y, Hernandez T, Yin $Y, X u Y$, Dixon RA. Switchgrass (Panicum virgatum) possesses a divergent family of Cinnamoyl CoA reductases with distinct biochemical properties. New Phytol. 2010;185(1):143-55.

37. Wingler A, Lea PJ, Leegood RC. Photorespiratory metabolism of glyoxylate and formate in glycine-accumulating mutants of barley and Amaranthus edulis. Planta. 1999;207(4):518-26.

38. Lu Y, Li Y, Yang Q, Zhang Z, Chen Y, Zhang S, Peng X. Suppression of glycolate oxidase causes glyoxylate accumulation that inhibits photosynthesis through deactivating Rubisco in rice. Physiol Plantarum. 2014;150(3):463-76.

39. Liepman $\mathrm{AH}$, Olsen $L$ J. Peroxisomal alanine : glyoxylate aminotransferase (AGT1) is a photorespiratory enzyme with multiple substrates in Arabidopsis thaliana. Plant J. 2001;25(5):487-98.

40. Eprintsev AT, Fedorin DN, Salnikov AV, Igamberdiev AU. Expression and properties of the glyoxysomal and cytosolic forms of isocitrate lyase in Amaranthus caudatus L. J Plant Physiol. 2015;181:1-8.

41. Zhang Z, Xu Y, Xie Z, Li X, He Z, Peng X. Association-dissociation of glycolate oxidase with catalase in rice: a potential switch to modulate intracellular $\mathrm{H}_{2} \mathrm{O}_{2}$ levels. Mol Plant. 2016;9(5):737-48.

42. Werner AK, Witte C. The biochemistry of nitrogen mobilization: purine ring catabolism. Trends Plant Sci. 2011;16(7):381-7.

43. Pick TR, Brautigam A, Schulz MA, Obata T, Fernie AR, Weber APM. PLGG1, a plastidic glycolate glycerate transporter, is required for photorespiration and defines a unique class of metabolite transporters. Proc Natl Acad Sci U S A. 2013;110(8):3185-90. 
44. Benning C. Evidence supporting a model of voltage-dependent uptake of auxin into Cucurbita vesicles. Planta. 1986;169(2):228-37.

45. Yoshida S, Forno DA, Cock JH, Gomez KA. Laboratory manual for physiological studies of rice. Manila: International Rice Research Institute; 1976.

46. Ma X, Zhang Q, Zhu Q, Wei L, Yan C, Rong Q, Wang B, Yang Z, Li H, Lin Y. A robust CRISPR/Cas9 system for convenient, high-efficiency multiplex genome editing in monocot and dicot plants. Mol Plant. 2015;8(8):1274-84.

47. Hiei Y, Ohta S, Komari T, Kumashiro T. Efficient transformation of rice (Oryza sativa L.) mediated by agrobacterium and sequence analysis of the boundaries of the T-DNA. Plant J. 1994;6(2):271-82.

48. Liu J, Cui L, Xie Z, Zhang Z, Liu E, Peng X. Two NCA1 isoforms interact with catalase in a mutually exclusive manner to redundantly regulate its activity in rice. BMC Plant Biol. 2019;19(1):105-10.

49. Lineweaver $\mathrm{H}$, Burk $\mathrm{D}$. The determination of enzyme dissociation constants. J Am Chem Soc. 1934;56(3):658-66.

50. Zhang Y, Su J, Duan S, Ao Y, Dai J, Liu J, Wang P, Li Y, Liu B, Feng D, Wang J, Wang $\mathrm{H}$. A highly efficient rice green tissue protoplast system for transient gene expression and studying light/chloroplast-related processes. Plant Methods. 2011;7(1):30.

51. Yoo S, Cho Y, Sheen J. Arabidopsis mesophyll protoplasts: a versatile cell system for transient gene expression analysis. Nat Protoc. 2007;2(7):1565-72.

\section{Publisher's Note}

Springer Nature remains neutral with regard to jurisdictional claims in published maps and institutional affiliations.

Ready to submit your research? Choose BMC and benefit from:

- fast, convenient online submission

- thorough peer review by experienced researchers in your field

- rapid publication on acceptance

- support for research data, including large and complex data types

- gold Open Access which fosters wider collaboration and increased citations

- maximum visibility for your research: over $100 \mathrm{M}$ website views per year

At $\mathrm{BMC}$, research is always in progress.

Learn more biomedcentral.com/submissions 